\title{
Article
}

\section{2-Month changes of muscle strength, body composition and physical activity in adults with dystrophinopathies}

Jaques, Matthew F, Onambele-Pearson, Gladys L, Reeves, Neil D, Stebbings, Georgina K, Dawson, Ellen A, Stockley, Rachel, Edwards, Bryn and Morse, Christopher L

Available at http://clok.uclan.ac.uk/34650/

Jaques, Matthew F, Onambele-Pearson, Gladys L, Reeves, Neil D, Stebbings, Georgina K, Dawson, Ellen A, Stockley, Rachel ORCID: 0000-0003-4441-6860, Edwards, Bryn and Morse, Christopher L (2020) 12-Month changes of muscle strength, body composition and physical activity in adults with dystrophinopathies. Disability \& Rehabilitation . ISSN 0963-8288

It is advisable to refer to the publisher's version if you intend to cite from the work. http://dx.doi.org/10.1080/09638288.2020.1808087

For more information about UCLan's research in this area go to

http://www.uclan.ac.uk/researchgroups/ and search for <name of research Group>.

For information about Research generally at UCLan please go to http://www.uclan.ac.uk/research/

All outputs in CLoK are protected by Intellectual Property Rights law, including Copyright law. Copyright, IPR and Moral Rights for the works on this site are retained by the individual authors and/or other copyright owners. Terms and conditions for use of this material are defined in the policies page. 


\section{2-Month changes of Muscle Strength, Body Composition and Physical Activity in adults with Dystrophinopathies}

Matthew F. Jacques ${ }^{1 *} \mathrm{PhD}$, Gladys L. Onambele-Pearson ${ }^{1} \mathrm{PhD}$, Neil D. Reeves ${ }^{1} \mathrm{PhD}$, Georgina K. Stebbings ${ }^{1} \mathrm{PhD}$, Ellen A Dawson ${ }^{2} \mathrm{PhD}$, Rachel C. Stockley ${ }^{3} \mathrm{PhD}$, Bryn Edwards ${ }^{4}$ BSc and Christopher I. Morse ${ }^{1} \mathrm{PhD}$.

\section{Research Centre for Musculoskeletal Science \& Sports Medicine, School of Healthcare} Science, Faculty of Science and Engineering, Manchester Metropolitan University, Manchester, United Kingdom. 2. Research Institute for Sport and Exercise Science, Liverpool John Moores University, Liverpool, United Kingdom 3. School of Nursing, University of Central Lancashire, Preston, United Kingdom. 4. The Neuromuscular Centre, Winsford, Cheshire, United Kingdom.

3


Abstract

Purpose. Muscular dystrophy (MD) is an umbrella term for muscle wasting conditions, for which longitudinal changes in function and body composition are well established in children with Duchenne (DMD), however changes in adults with DMD and Beckers (BMD), respectively, remain poorly reported. This study aims to assess 12-month changes in lowerlimb strength, muscle size, body composition and physical activity in adults with Muscular Dystrophy (MD).

Methods. Adult males with Duchenne MD (DMD; N = 15) and Beckers MD (BMD; N = 12) were assessed at baseline and 12-months for body composition (Body fat and lean body mass (LBM)), Isometric maximal voluntary contraction (Knee-Extension (KEMVC) and PlantarFlexion (PFMVC)) and physical activity (tri-axial accelerometry).

Results. 12-month change in strength was found as -19\% (PFMVC) and -14\% (KEMVC) in DMD. 12-month change in strength in BMD, although non-significant, was explained by physical activity $\left(\mathrm{R}^{2}=.532-.585\right)$. Changes in LBM (DMD) and body fat (BMD) were both masked by non-significant changes in body mass.

Discussion. 12-month changes in adults with DMD appear consistent with paediatric populations. Physical activity appears important for muscle function maintenance. Specific monitoring of body composition, and potential co-morbidities, within adults with MD is highlighted. 

resulting in progressive muscle weakness and declining muscle mass [1]. Unlike many other forms of MD, which affect a variety of different proteins associated with the sarcolemma [2], DMD and BMD are unique in that they are both affected by impairment of the same protein, named Dystrophin [3, 4]. DMD results from an absent or non-functioning dystrophin protein, therefore is more progressive, with loss of ambulation by the age of 12 [5, 6]. BMD in comparison is caused by a partially functioning dystrophin protein, therefore a slower and more variable form of MD, with the loss of ambulation in adulthood [5, 6]. Despite the well acknowledged genetic understanding of these conditions [3, 4, 7-9], and a breadth of research assessing health and function in children with DMD [10-17], basic understanding of the progression of these conditions and impact on function and health measures remains minimal in adult populations [18].

Lower limb muscle strength has historically been a key outcome measure reported in MD [1925], with assessment using direct measures (either objectively using dynamometers or through subjective assessments such as manual muscle testing (MMT)) or indirect measures, such as 
sit-to-stand or 10m walk time [19, 22, 26-29]. Longitudinal strength change in BMD has only been described through MMT assessment of knee extension strength (KEMVC) however, showing annual declines of $1.2 \%$ [28]. More recently, the current authors demonstrated that in adults with MD, variance in KEMVC and functional measures could be explained by accelerometer determined physical activity (PA)[19]. It is therefore important to understand the rate of strength decline in adults with BMD, but also to assess the impact of PA on strength.

Cross-sectional and natural history studies by comparison are more common within children with DMD [20, 21, 23, 30, 31]. Indeed, muscle weakness is typically identified during childhood in DMD, with impaired gait an early indicator of DMD [32, 33]. Subjective methods of MMT or Medical research council scales (MRC\%) have reported annual declines of KEMVC as $4-5 \%$ and $1.2-2 \%$ in ambulant (5-13 years) and non-ambulant (13-24 years) children with DMD, respectively [27, 34, 35]. Objective measures such as dynamometers however have identified, annual declines of KEMVC as $15 \%$ in children with DMD (8-12 years) [36]. Despite lower limb muscle strength having limited clinical relevance in adults with DMD, it remains essential that a comprehensive understanding of the progression of DMD in this older, unreported age group is developed, in order to develop a life-long understanding of condition progression, provide comparative norms using relevant and accessible methods, as well as to provide comparisons for future longitudinal assessments of steroid or gene therapy studies which may be relevant to this group $[18,37]$.

Strength and function have been associated with Lean Body Mass (LBM) in children with DMD $[38,39]$. While pseudohypertrophy (increased muscle size without relative increase in strength) of the calves is well documented in children and adolescents with DMD [31, 40, 41], recent research suggests it may not persist in adults with DMD however [19, 42]. Furthermore, the pre-disposition of impaired muscular, respiratory and cardiac systems to ill health can be placed under further pressure by increased sedentary behaviour [19], resulting in greater fat 
mass, which has previously been cited as a common co-morbidity in adults with MD [43, 44], and reported as higher in non-ambulant than ambulant adults with BMD [45]. Continual assessment, and understanding, of body composition changes of both lean and fat mass is essential, for not only their implications on function, but also the much broader impacts on health and wellbeing $[13,46]$.

This study aims to: 1) Quantify changes, from a one year follow up, in body composition, muscle strength, muscle size and physical activity levels in adults with DMD and BMD; and 2) Identify the impact of changes in physical activity on body composition and muscle strength.

The authors hypothesise that declines will be greater in DMD than BMD, although still evident in both conditions, for lower limb strength, muscle size and LBM. In addition, PA may account for some of the variance in lower limb strength change in BMD, but not DMD.

\section{Materials and Methods}

This study comprised of adult male volunteers with DMD ( $\mathrm{n}=15)$ and BMD $(n=12)$. All participants were recruited from, and tested at, The Neuromuscular Centre (Winsford, UK). No participants were habitually taking part in a structured training programme, however all were receiving weekly, bi-weekly or monthly physiotherapy treatment, consisting of passive stretching, along with access to low intensity cardiovascular exercise equipment (monthly frequency of physiotherapy for DMD $=4(1-4), \mathrm{BMD}=2$ (1-2) expressed as Median (range). Ethical approval was obtained through the Manchester Metropolitan University Ethics Committee, and all participants signed informed consent forms prior to participation. All procedures complied with the latest edition of the World Medical Association Declaration of Helsinki [47].

All method protocols, data presentation and reliability, have been reported previously [19], where they can be read in full. A brief overview of each method has been presented below. 


\section{Procedures}

All participants undertook Baseline and $12 \pm 1$ month follow up testing. Testing involved functional and morphological tests, which was followed by a 7-day PA assessment, using wristworn three-dimensional accelerometers, worn 24 hours a day. The same equipment was used for all participants and due to the high level of contractures present in some participants; all participants were assessed in a seated position to ensure consistency.

\section{Sample Size}

In order to determine the sample size required to provide a representative sample for 12 month changes in adult populations of DMD, statistical a Priori power calculations were performed using G*Power 3.1.9.2 software (Franz Faul, Universitat Kiel, Germany). For this calculation, alpha was set a 0.05 and beta at 0.80 . The DMD sample size was calculated to show a $10 \%$ change in muscle strength score consistent with the natural history group previously reported by Mendell et al. [34]. This method calculated an adequate adult DMD sample size of $n=15$. For BMD, due to the lack of extant data for a Priori calculation to be performed, it was deemed that the power calculation for BMD participants in clinical trials $(n=15)$ by Bello et al. [7] was appropriate.

\section{Anthropometry}

All participants were weighed in a digital seated scales system (6875, Detecto, Webb City, Mo, USA). Slings, shoes, splints etc. were weighed separately and subtracted from the gross weight. All participants' height was calculated as point-to-point of arm span (index finger, elbow, shoulder and across midline) to replicate the method used on non-ambulatory participants [45, 48].

\section{Body Composition}

Body composition measures of body fat and LBM were measured using Bioelectrical Impedance (BIA) in a fasted state following a 12 hour fast, with adhesive electrodes placed on 
the right hand and foot. BIA has been promoted as a measure for change in fat and LBM over time in a dystrophic population [16].

Lean Body Mass was determined by the following equation:

$$
\operatorname{LBM}(\mathrm{Kg})=\text { Body Mass }(\mathrm{Kg})-\text { Fat Mass }(\mathrm{Kg})
$$

Body Mass Index (BMI) was calculated using the following equation [49]:

$$
\operatorname{BMI}\left(\frac{K g}{m^{2}}\right)=\operatorname{Body} \operatorname{Mass}(K g) \div \operatorname{Height}^{2}\left(m^{2}\right)
$$

\section{Muscle Strength}

Due to the high levels of contractures present in adults with DMD, strength testing protocols were designed to be completed on the most mechanically limited participants, and replicated on all others. Therefore, isometric plantar flexion maximal voluntary contraction (PFMVC) and KEMVC force was recorded using a load cell, with all participants in a seated position replicative of quantitative muscle testing [31]. The load cell was calibrated using a known load of $500 \mathrm{~g}-5 \mathrm{~kg}$, in $500 \mathrm{~g}$ increments, prior to every strength testing session. MVC measures all took place with the participant seated, with hip and knee angles maintained at $90^{\circ}$, for which non-ambulant participants remained in their manual/power wheelchair. For KEMVC, a strap was tightly fastened around the participant's ankle, and attached perpendicularly to the load cell, which was fastened to a weighted support bar. For PFMVC the participants foot was attached to a footplate, with the load cell attached underneath. PFMVC measures were taken from $0^{\circ}$ (neutral position), or as close to neutral as possible due to equinus deformity evident in DMD [50]. For PFMVC the practitioner provided the resistive force to ensure an isometric contraction, and all measures of force were normalised for gravity. Three trials were performed for PFMVC and KEMVC respectively, with extended breaks of 1 minute between trials due to the increased fatigue associated with MD [51]. Force $(\mathrm{N})$ was converted to torque $(\mathrm{N} \cdot \mathrm{m})$ by 
multiplying the force measurement by the moment arm from the axis of rotation (knee or ankle) to the point of force measurement (the strap height on the shin, or ball of the foot). PFMVC and KEMVC measures have been presented as torque (N.m).

This method has been shown to be highly reliable for both PFMVC and KEMVC in adults with DMD (Within Day ICC: 0.98 and 0.99; Between Day ICC: 0.98 and 0.99) and BMD (Within Day ICC: 0.91 and 0.99; Between Day ICC: 0.83 and 0.99) [19].

\section{Muscle Size Assessment}

Gastrocnemius Medialis (GM) anatomical cross sectional area (ACSA) was measured using transverse ultrasound scans (7.5-MHz linear array probe) at $50 \%$ of muscle length, consistent with the muscle length at which the largest ACSA occurs [52]. Echoabsorptive tape (Transpore, $3 \mathrm{M}$, USA) was used to project shadows on the ultrasound image during recording to provide a positional reference. From which still images were captured then recreated into a single image offline (Graphic Image Manipulation Program, GIMP Development) using the shadows from echoabsorptive tape, muscle markers and aponeurosis of the muscle. The ACSA was then measured using digitising software (ImageJ 1.45, National Institute of Health, USA). Further details can be found in our previous reports of GM ACSA in MD [42, 45]. This method of ACSA assessment has been reported previously as reliable (0.998) and valid (0.999) in comparison to Magnetic Resonance Imaging (MRI) [53].

It is important to note that this method measures the area within the fascia of the muscle boundaries only, it cannot differentiate muscle or fibrous tissue (more commonly recognised as fat fraction) as seen in MRI $[31,54-56]$. Therefore, GM ACSA is a method of assessing psuedohypertrophy only, and not muscle quality or contractile capacity. 
Nine ambulant BMD participants performed a $10 \mathrm{~m}$ walk test, one participant however lost ambulation during the one year follow up period, therefore data is presented of the 8 participants that completed both the Baseline and 12 months testing. The $10 \mathrm{~m}$ walk was performed on an even surface, and is a common measure of function within neuromuscular conditions $[36,57]$. All participants started in a standing position and were instructed to walk as quickly and safely as they could, with the time recorded from the verbal instruction of "Go" from the practitioner, to the point of crossing the finish line. Walking aids were permitted if required. Participants $10 \mathrm{~m}$ walk time were recorded as early in the day as possible to limit the effect of fatigue, with the 12-month measure taking place at the same time.

\section{Physical Activity}

Daily PA was monitored over a consecutive 7-day period using a wrist-worn tri-axial accelerometer (GENEActiv, Kimbolton, Cambs, United Kingdom). Monitors were initiated to collect data at $100 \mathrm{~Hz}$, worn for 24 hours a day on the preferred wrist of participants and worn continuously for 7 days [58]. Upon completion of 7-day monitoring, data is downloaded into .bin files, converted to 60s epoch .csv files using the GENEActiv PC Software (Version 2.1). 60s epoch data files were then entered into an open source Excel macro (v2, Activinsights Ltd.) [59]. GENEActiv monitors have shown high validity for the measurement of both PA and SB (Pearon's $r=0.79-0.98)[59,60]$. PA is presented as a percentage of time spent sedentary $(\mathrm{SB} \%)$ or total time spent physically active $\left(\mathrm{TPA}^{\mathrm{mins}}\right)[19]$.

\section{Functional Status}

All participants functional status was assessed by an experienced neuromuscular 222 physiotherapist using the Swinyard Severity Classification scale [61]. The Swinyard Severity 223 Classification grades function and ability to carry out activities of daily living from Stage 1 "mild abnormalities in gait, able to climb stairs without assistance", to Stage 8 "Unable to sit 
without considerable support, requires maximal assistance for activities of daily living". The Swinyard Severity Scale has been used extensively in MD research [62-64], and shown to be highly correlated with fraction of lower limb muscle mass in DMD [54].

\section{Statistical Analyses}

229

230

All analyses were performed using IBM SPSS Statistics v21 software with a critical level of statistical significance set at 5\% and all data presented as mean (SD), except for Functional Status which is presented as Median (Range). We have previously published between group differences for baseline measures [19], with the present study interested in differences from baseline-12 months, therefore statistical analysis has been performed on baseline to 12 month changes only (within group), with baseline values presented for clarity. Test for parametricity were performed upon all variables, for repeated measures in DMD, body mass, height, BMI, Lean Mass and PFMVC were parametric, and all other variables were non-parametric. For BMD height, body fat, Lean Mass, GM ACSA, PFMVC, SB\% and TPA ${ }^{\text {mins }}$ were parametric, all other variables were non-parametric. Respiratory, Gastrostomy and Ambulatory statuses are presented as a characteristic and no statistical analysis was performed on it.

For repeated measures, Paired T-tests and Wilcoxon signed rank tests, for parametric and nonparametric respectively, were used to identify changes, with a Bonferroni correction. Where relevant, comparisons are presented with $\mathrm{P}$ values, the relative change $(\%)$ from baseline and 95\% Confidence Intervals.

Stepwise Multiple Linear Regression was used to identify the best predictors of PFMVC change from GM ACSA Change, LBM Change and Baseline PFMVC. Linear, Quadratic and Cubic regressions are used to best model changes in body composition and muscle strength in relation to age and changes in TPA ${ }^{\text {mins }}$, with the best fit model presented. 


\section{Month Changes}

Compared to baseline, 12 month PFMVC and KEMVC decreased in DMD by $19 \%(\mathrm{P}=0.002)$ and $14 \%(\mathrm{P}=0.003)$, respectively. Compared to baseline, 12 month LBM and GM ACSA decreased by $5 \%(\mathrm{P}=0.002)$ and $8 \%(\mathrm{P}=0.012)$ respectively, in DMD. No other differences were identified between baseline and 12 months for measures of anthropometrics, body composition or muscle size for DMD (table 1, P>0.05).

\section{$B M D$}

There was no difference in KEMVC or PFMVC compared to baseline in BMD ( $\mathrm{P}>0.05)$.

Compared to baseline $10 \mathrm{~m}$ walk time increased in ambulant $\mathrm{BMD}$ by $13 \%(\mathrm{P}=0.005)$. No other differences were identified between baseline and 12 months for any other measures (table 1).

Compared to baseline there was no significant change in GM ACSA or LBM in BMD (P>0.05). In BMD, compared to baseline, Body Fat increased by $4 \%(\mathrm{P}=0.009)$ after 12 months. One BMD participant lost ambulation between baseline and 12 months. No other differences were identified between baseline and 12 months for measures of anthropometric, body composition or muscle size for BMD (table 2, $\mathrm{P}>0.05$ ).

\section{Regressions}

268 Stepwise Multiple Linear Regression identified a model containing Baseline PFMVC, GM 269 ACSA change and LBM Change best predicted PFMVC Change in DMD $\left(\mathrm{R}^{2}=0.582\right.$, $\mathrm{P}=0.019)$.

271 No relationship was identified for DMD using any regression model for age or TPA ${ }^{\text {mins }}$ change

272 with change in PFMVC, KEMVC, LBM or body fat $(\mathrm{P}>0.05)$. No relationships were identified 
for either DMD or BMD using any regression model for age with change in PFMVC, KEMVC, LBM or body fat, or TPA ${ }^{\text {mins }}$ change with change in LBM or body fat $(\mathrm{P}>0.05)$.

In BMD quadratic polynomial regressions best identified relationships for TPA ${ }^{\text {mins }}$ change with PFMVC change $\left(\mathrm{R}^{2}=.585, \mathrm{P}=0.019\right.$, figure $\left.1 \mathrm{~A}\right)$ and KEMVC change $\left(\mathrm{R}^{2}=0.532, \mathrm{P}=0.033\right.$, figure 1B). No relationships were identified in DMD using any regression model for TPA ${ }^{\text {mins }}$ PFMVC change or KEMVC change $(\mathrm{P}>0.05)$.

\section{[Figure 1 Here]}

\section{Discussion}

The present study reports 12 month changes in lower limb muscle strength, muscle size and body composition in adults with BMD and DMD. 12-month changes in lower limb function have been identified using objective measures of muscle strength in adults with DMD and BMD. After 12 months, LBM, GM ACSA, PFMVC and KEMVC decreased in DMD, whereas in BMD there was no change in any measure, other than body fat which increased. Although there was no significant decrease in strength within BMD, the variance in the 12-month change of PFMVC and KEMVC was partially attributable to the variance in physical activity change over the same period.

The $14 \%$ decline in KEMVC in adults with DMD in the present study is consistent with the $15 \%$ decline previously reported over a similar timeframe in children with DMD [36]. These declines in KEMVC are in contrast to the $2 \%$ and $1.2 \%$ declines reported in non-ambulant children and adolescents with DMD, respectively $[35,36]$. This discrepancy can be attributed to the greater sensitivity of the methods used in the present study to quantify changes in KEMVC, rather than subjective measures of MMT or MRC\% $[65,66]$. In adults with BMD we observed no significant change in KEMVC or PFMVC, likely due to greater variance 
associated with the condition, however the quantified declines of $14 \%$ KEMVC and $7 \%$

PFMVC remain noteworthy.

The increase in body fat in BMD $(+4 \%)$ in the present study appears consistent with our previous research in which excess weight gain was identified as an issue in BMD, especially in non-ambulant individuals [45]. The relative increase in body fat in BMD compared to DMD may be due to the fact that BMD maintain a greater level of function and physical independence [67], compared to DMD [48] who require assistance in the preparation and consumption of food. Monitoring and management of food intake may be easier and more structured in DMD [68], particularly given 4/15 participants in the current study consumed via PEG. The stable body mass in both DMD and BMD did however mask changes in body composition, with decreased LBM in DMD and increased body fat in BMD. Therefore reaffirming the need for body composition monitoring in these conditions [16].

Adults with BMD that maintained or increased PA levels showed a relative increase or maintenance of muscle strength compared to those that decreased PA levels. Increased PA has previously been attributed to decelerating fatty infiltration of muscles in FSHD [69]. Based on the present relationship between PA and declines in muscle strength, it seems reasonable to suggest interventions that increase PA in adults with BMD may benefit muscle strength, while potentially also alleviating some concerns around changes in fat mass identified in the present study. Future work needs to investigate the benefits of increasing PA, and to further identify psycho-somatic and/or social barriers and facilitators of PA and patterns of SB in this population [70].

\section{Study Limitations}

318 The present study has two main limitations, the first being the sample size. Whilst the sample size recruited is aligned with those identified during the a Priori power calculations (See 
The present study however does report on longitudinal changes in function and health in a previously unreported sample, adults with DMD [37], and utilises outcome measures that are more sensitive to previous methods. Differences were identified within the present DMD sample size of $n=15$, while a Post Hoc calculation for adults with BMD using data from the present study identifies $\mathrm{n}=15$ required for future studies monitoring lower limb muscle strength. Whilst the recruited BMD sample size in the present study is slightly under-powered, it is considerably larger than that reported previously in natural history studies on adults with BMD [28], and contributes significantly to the currently limited longitudinal data in adults with BMD.

Secondly, the present study is limited to 12 months monitoring only, which is comparably shorter than some previous studies [28, 35], however consistent with many previous longitudinal studies in children with DMD [25, 72-74]. The 12 month sample period was long enough however to identify specific changes in LBM (DMD), body fat (BMD), GM ACSA (DMD), PFMVC (DMD), KEMVC (DMD) and 10m walk time (BMD). Regardless, this identification of differences in function and health within a 12 month time period is an important finding itself, and further emphasises the need for continuous health and function monitoring and management in these conditions.

All DMD participants will have received some form of steroid treatment through childhood and adolescence. Whereby steroid treatment typically stops upon full-time wheelchair use. Given the data collection from a non-NHS organisation, it is beyond the scope of the present investigation to gain historical steroid treatment and dosage information. Therefore, all data has been presented with the caveat that DMD participants will have historically received steroid treatment, however it should be noted that none were currently receiving steroid treatment. 
345 Whilst it is important to further understand the progression of these conditions in what has been previously described as an "unforeseen population" [37], further mechanistic insight is required. Primarily, the reductions in strength in the current study are likely attributed to progressive fat fraction within the muscle, synonymous with the condition [20]. Future research should assess the progression of tissue changes in adults with DMD, however the reduction in GM ACSA in the current study appears consistent with previous hypothesis' that muscle size becomes more representative of contractile tissue quantity in adulthood [42], with the end of the inflammatory induced appearance of psuedohypertrophy. In addition, further understanding of physical behaviours in adults with BMD is required, especially those who retain some form of ambulation, given the present findings on body composition, and previous work demonstrated positive effects of increased step count on contractile tissue in adults with Fascioscapulohumeral MD [75]. More broadly, evidence based nutritional guidelines, with specifics guidance for differing classifications and functional status are required to best manage energy balance and reduce additional strains on health.

\section{Conclusion}

In conclusion, the present data describes natural history changes in body composition, strength and physical activity in adults with DMD and BMD. Changes in DMD appear consistent with the understanding of the condition, with 14-19\% weaker PFMVC and KEMVC, consistent with paediatric populations $[16,36,42]$. Change in DMD PFMVC was best explained by changes in LBM, GM ACSA and Baseline PFMVC. Within BMD, 12 month changes in PFMVC and KEMVC although not significant, were explained by change in minutes of physical activity. Changes in LBM in DMD and body fat in BMD were both masked by non-significant changes in body mass, furthering the need for specific monitoring of body composition to reduce the development of potential co-morbidities. 
372 No potential conflict of interest is reported by the authors

\section{Data Availability}

374 Data is available upon request to the Author.

375

376

377

378

379

380

\section{References}

1. Deconinck, N. and B. Dan, Pathophysiology of duchenne muscular dystrophy: current hypotheses. Pediatric neurology, 2007. 36(1): p. 1-7.

2. Huml, R.A., Muscular Dystrophy: A Concise Guide. 2015: Springer.

3. Bushby, K.M.D., et al., The clinical, genetic and dystrophin characteristics of Becker muscular dystrophy. Journal of neurology, 1993. 240(2): p. 105-112.

4. Hoffman, E.P., R.H. Brown, and L.M. Kunkel, Dystrophin: the protein product of the Duchenne muscular dystrophy locus. Cell, 1987. 51(6): p. 919-928.

5. Emery, A.E.H., Population frequencies of inherited neuromuscular diseases-a world survey. Neuromuscular disorders, 1991. 1(1): p. 19-29.

6. Emery, A.E.H., The muscular dystrophies. The Lancet, 2002. 359(9307): p. 687-695.

7. Bello, L., et al., Functional changes in Becker muscular dystrophy: implications for clinical trials in dystrophinopathies. Scientific reports, 2016. 6: p. 32439.

8. Ervasti, J.M., et al., Deficiency of a glycoprotein component of the dystrophin complex in dystrophic muscle. Nature, 1990. 345(6273): p. 315-319.

9. Morandi, L., et al., Dystrophin analysis in Duchenne and Becker muscular dystrophy carriers: correlation with intracellular calcium and albumin. Annals of neurology, 1990. 28(5): p. 674679.

10. Inkley, S.R., F.C. Oldenburg, and P.J. Vignos, Pulmonary function in Duchenne muscular dystrophy related to stage of disease. The American journal of medicine, 1974. 56(3): p. 297306.

11. Brooke, M.H., et al., Duchenne muscular dystrophy Patterns of clinical progression and effects of supportive therapy. Neurology, 1989. 39(4): p. 475-475.

12. Bakker, J.P.J., et al., Predictive factors of cessation of ambulation in patients with Duchenne muscular dystrophy. American journal of physical medicine \& rehabilitation, 2002. 81(12): p. 906-912.

13. Davidson, Z.E. and H. Truby, A review of nutrition in Duchenne muscular dystrophy. Journal of human nutrition and dietetics, 2009. 22(5): p. 383-393.

14. Jones, D.A., et al., Size and composition of the calf and quadriceps muscles in Duchenne muscular dystrophy. A tomographic and histochemical study. Journal of the neurological sciences, 1983. 60(2): p. 307-22.

15. Kohler, M., et al., Quality of life, physical disability, and respiratory impairment in Duchenne muscular dystrophy. American journal of respiratory and critical care medicine, 2005. 172(8): p. 1032-1036.

16. Mok, E., et al., Assessing change in body composition in children with Duchenne muscular dystrophy: anthropometry and bioelectrical impedance analysis versus dual-energy $X$-ray absorptiometry. Clinical nutrition, 2010. 29(5): p. 633-638.

17. Henricson, E., et al., The 6-minute walk test and person-reported outcomes in boys with duchenne muscular dystrophy and typically developing controls: longitudinal comparisons and clinically-meaningful changes over one year. PLoS currents, 2013. 5. 
18. Bettolo, C.M., et al., Adult Duchenne population: A growing population. Neuromuscular Disorders, 2016. 26: p. S126.

19. Jacques, M.F., et al., Relationships between muscle size, strength, and physical activity in adults with muscular dystrophy. Journal of Cachexia, Sarcopenia and Muscle, 2018. 9(6): p. 1042-1052.

20. Akima, H., et al., Relationships of thigh muscle contractile and non-contractile tissue with function, strength, and age in boys with Duchenne muscular dystrophy. Neuromuscular Disorders, 2012. 22(1): p. 16-25.

21. Lerario, A., et al., Quantitative muscle strength assessment in duchenne muscular dystrophy: longitudinal study and correlation with functional measures. BMC neurology, 2012. 12(1): p. 91.

22. Alfano, L.N., et al., Correlation of knee strength to functional outcomes in becker muscular dystrophy. Muscle \& nerve, 2013. 47(4): p. 550-554.

23. Bendixen, R.M., et al., Participation in daily life activities and its relationship to strength and functional measures in boys with Duchenne muscular dystrophy. Disability and rehabilitation, 2014. 36(22): p. 1918-1923.

24. Alemdaroğlu, l., et al., Different types of upper extremity exercise training in Duchenne muscular dystrophy: Effects on functional performance, strength, endurance, and ambulation. Muscle \& nerve, 2015. 51(5): p. 697-705.

25. Seferian, A.M., et al., Upper limb strength and function changes during a one-year follow-up in non-ambulant patients with Duchenne muscular dystrophy: an observational multicenter trial. PLoS One, 2015. 10(2): p. e0113999.

26. Kilmer, D.D., R.T. Abresch, and J.W.M. Fowler, Serial manual muscle testing in Duchenne muscular dystrophy. Archives of physical medicine and rehabilitation, 1993. 74(11): p. 11681171.

27. McDonald, C., et al., Profiles of neuromuscular diseases: Duchenne muscular dystrophy. American journal of physical medicine \& rehabilitation, 1995. 74(5): p. S93.

28. McDonald, C.M., et al., Profiles of neuromuscular diseases: Becker's muscular dystrophy. American journal of physical medicine \& rehabilitation, 1995. 74(5): p. S104.

29. McDonald, C.M., et al., The 6-minute walk test and other clinical endpoints in duchenne muscular dystrophy: Reliability, concurrent validity, and minimal clinically important differences from a multicenter study. Muscle \& nerve, 2013. 48(3): p. 357-368.

30. Batra, A., et al., Two-Year Longitudinal Changes in Lower Limb Strength and Its Relation to Loss in Function in a Large Cohort of Patients With Duchenne Muscular Dystrophy. American journal of physical medicine \& rehabilitation, 2018. 97(10): p. 734-740.

31. Wokke, B.H., et al., Quantitative MRI and strength measurements in the assessment of muscle quality in Duchenne muscular dystrophy. Neuromuscular Disorders, 2014. 24(5): p. 409-416.

32. D'Angelo, M.G., et al., Gait pattern in Duchenne muscular dystrophy. Gait \& posture, 2009. 29(1): p. 36-41.

33. Hsu, J.D. and J. Furumasu, Gait and posture changes in the Duchenne muscular dystrophy child. Clinical orthopaedics and related research, 1993. 288: p. 122-125.

34. Mendell, J.R., et al., Randomized, double-blind six-month trial of prednisone in Duchenne's muscular dystrophy. New England Journal of Medicine, 1989. 320(24): p. 1592-1597.

35. Steffensen, B.F., et al., Physical capacity in non-ambulatory people with Duchenne muscular dystrophy or spinal muscular atrophy: a longitudinal study. Developmental medicine and child neurology, 2002. 44(9): p. 623-632.

36. McDonald, C.M., et al., THE 6-minute walk test and other endpoints in Duchenne muscular dystrophy: Longitudinal natural history observations over 48 weeks from a multicenter study. Muscle \& nerve, 2013. 48(3): p. 343-356. 
37. Rahbek, J., et al., Adult life with Duchenne muscular dystrophy: observations among an emerging and unforeseen patient population. Pediatric Rehabilitation, 2005. 8(1): p. 17-28.

38. Palmieri, G.M.A., et al., Assessment of whole body composition with dual energy X-ray absorptiometry in Duchenne muscular dystrophy: Correlation of lean body mass with muscle function. Muscle \& Nerve: Official Journal of the American Association of Electrodiagnostic Medicine, 1996. 19(6): p. 777-779.

39. Skalsky, A.J., et al., Assessment of regional body composition with dual-energy X-ray absorptiometry in Duchenne muscular dystrophy: Correlation of regional lean mass and quantitative strength. Muscle \& nerve, 2009. 39(5): p. 647-651.

40. Beenakker, E.A.C., et al., Quantitative assessment of calf circumference in Duchenne muscular dystrophy patients. Neuromuscular Disorders, 2002. 12(7): p. 639-642.

41. Vohra, R.S., et al., Magnetic resonance assessment of hypertrophic and pseudo-hypertrophic changes in lower leg muscles of boys with duchenne muscular dystrophy and their relationship to functional measurements. PloS one, 2015. 10(6): p. e0128915.

42. Morse, C.I., et al., Gastrocnemius medialis muscle architecture and physiological cross sectional area in adult males with Duchenne muscular dystrophy. J Musculoskelet Neuronal Interact, 2015. 15(2): p. 154-160.

43. Zanardi, M.C., et al., Body composition and energy expenditure in Duchenne muscular dystrophy. European journal of clinical nutrition, 2003. 57(2): p. 273-278.

44. Bostock, E.L., et al., Impaired glucose tolerance in adults with duchenne and becker muscular dystrophy. Nutrients, 2018. 10(12): p. 1947.

45. Jacques, M.F., et al., Resting Energy Expenditure in Adults with Becker's Muscular Dystrophy. PloS one, 2017. 12(1): p. e0169848.

46. Hogan, S.E., Body composition and resting energy expenditure of individuals with Duchenne and Becker muscular dystrophy. Canadian Journal of Dietetic Practice and Research, 2008. 69(4): p. 208-212.

47. World Medical, A., World Medical Association Declaration of Helsinki: ethical principles for medical research involving human subjects. Jama, 2013. 310(20): p. 2191.

48. Morse, C.I., et al., The cardiorespiratory response and physiological determinants of the assisted 6-minute handbike cycle test in adult males with muscular dystrophy. Muscle \& nerve, 2018. 58(3): p. 427-433.

49. McCabe, M.P., L.A. Ricciardelli, and P. Parent, BODY MASS INDEX. Eating Disorders: An Encyclopedia of Causes, Treatment, and Prevention, 2013. 34: p. 90.

50. Williams, E.A., et al., The management of equinus deformity in Duchenne muscular dystrophy. The Journal of bone and joint surgery. British volume, 1984. 66(4): p. 546-550.

51. Sharma, K.R., M.A. Mynhier, and R.G. Miller, Muscular fatigue in Duchenne muscular dystrophy. Neurology, 1995. 45(2): p. 306-310.

52. Fukunaga, T., et al., Physiological cross-sectional area of human leg muscles based on magnetic resonance imaging. Journal of Orthopaedic Research, 1992. 10(6): p. 926-934.

53. Reeves, N.D., C.N. Maganaris, and M.V. Narici, Ultrasonographic assessment of human skeletal muscle size. European journal of applied physiology, 2004. 91(1): p. 116-118.

54. Liu, M., N. Chino, and T. Ishihara, Muscle damage progression in Duchenne muscular dystrophy evaluated by a new quantitative computed tomography method. Archives of physical medicine and rehabilitation, 1993. 74(5): p. 507-514.

55. Løkken, N., et al., Contractile properties are disrupted in Becker muscular dystrophy, but not in limb girdle type 2I. Annals of Neurology, 2016.

56. Willcocks, R.J., et al., Longitudinal measurements of MRI-T2 in boys with Duchenne muscular dystrophy: effects of age and disease progression. Neuromuscular Disorders, 2014. 24(5): p. 393-401.

57. Fowler, E.G., et al., Longitudinal Community Walking Activity in Duchenne Muscular Dystrophy. Muscle \& Nerve, 2017. 57(3): p. 401-406. 
58. Dillon, C.B., et al., Number of days required to estimate habitual activity using wrist-worn GENEActiv accelerometer: A cross-sectional study. PloS one, 2016. 11(5): p. e0109913.

59. Esliger, D.W., et al., Validation of the GENEA Accelerometer. Medicine and Science in Sports and Exercise, 2011. 43(6): p. 1085-1093.

60. Phillips, L.R.S., G. Parfitt, and A.V. Rowlands, Calibration of the GENEA accelerometer for assessment of physical activity intensity in children. Journal of science and medicine in sport, 2013. 16(2): p. 124-128.

61. Swinyard, C.A., G.G. Deaver, and L. Greenspan, Gradients of functional ability of importance in rehabilitation of patients with progressive muscular and neuromuscular diseases. Archives of physical medicine and rehabilitation, 1957. 38(9): p. 574-579.

62. Melacini, P., et al., Cardiac and respiratory involvement in advanced stage Duchenne muscular dystrophy. Neuromuscular disorders, 1996. 6(5): p. 367-376.

63. Hunsaker, R.H., et al., Cardiac function in Duchenne's muscular dystrophy: Results of 10-year follow-up study and noninvasive tests. The American journal of medicine, 1982. 73(2): p. 235-238.

64. Yamada, Y., et al., Long-term follow-up of dysphagia in adult patients with Duchenne muscular dystrophy. European Journal of Paediatric Neurology, 2018. 22(5): p. 786-790.

65. Hogrel, J.-Y., et al., Development of a French isometric strength normative database for adults using quantitative muscle testing. Archives of physical medicine and rehabilitation, 2007. 88(10): p. 1289-1297.

66. Cuthbert, S.C. and G.J. Goodheart, On the reliability and validity of manual muscle testing: $a$ literature review. Chiropractic \& osteopathy, 2007. 15(1): p. 4.

67. Lue, Y.-J., et al., Measurement of the functional status of patients with different types of muscular dystrophy. The Kaohsiung journal of medical sciences, 2009. 25(6): p. 325-333.

68. Pane, M., et al., Feeding problems and weight gain in Duchenne muscular dystrophy. European journal of paediatric neurology, 2006. 10(5): p. 231-236.

69. Ferguson, M.R., et al., Quantitative MRI reveals decelerated fatty infiltration in muscles of active FSHD patients. Neurology, 2016. 87(16): p. 1746-1746.

70. Phillips, M., N. Flemming, and K. Tsintzas, An exploratory study of physical activity and perceived barriers to exercise in ambulant people with neuromuscular disease compared with unaffected controls. Clinical Rehabilitation, 2009. 23(8): p. 746-755.

71. Pane, M., et al., Long term natural history data in ambulant boys with Duchenne muscular dystrophy: 36-month changes. PLoS One, 2014. 9(10): p. e108205.

72. Pane, M., et al., 6 Minute walk test in Duchenne MD patients with different mutations: 12 month changes. PloS one, 2014. 9(1): p. e83400.

73. Mazzone, E., et al., Functional changes in Duchenne muscular dystrophy A 12-month longitudinal cohort study. Neurology, 2011. 77(3): p. 250-256.

74. Bonati, U., et al., Quantitative muscle MRI: a powerful surrogate outcome measure in Duchenne muscular dystrophy. Neuromuscular Disorders, 2015. 25(9): p. 679-685.

75. Janssen, B., et al., Quantitative MRI reveals decelerated fatty infiltration in muscles of active FSHD patients. Neurology, 2016. 86(18): p. 1700-1707.

Table 1. 12 Month changes in body composition, muscle size, lower limb strength and physical activity in Adults with DMD.

\begin{tabular}{|c|c|c|c|c|}
\hline \multicolumn{5}{|c|}{ DMD } \\
\hline & Baseline & 12-Months & $\%$ Change & $95 \% \mathrm{CI}$ \\
\hline $\mathbf{N}$ & & & & \\
\hline $\begin{array}{l}\text { Functional Status } \\
\text { Ambulatory Status }\end{array}$ & $8(8-8)$ & $8(8-8)$ & - & - \\
\hline
\end{tabular}


No Walking

Support

Walking Support

Manual Wheelchair

Electric Wheelchair

Respiratory

Support

$\begin{array}{ccc}\text { Night-time only (\%) } & 13 / 15 & 13 / 15 \\ 24 / 7(\%) & 2 / 15 & 2 / 15 \\ \text { PEG (\%) } & 4 / 15 & 4 / 15\end{array}$

Age (years) $\quad 24.2 \pm 6.1 \quad 25.2 \pm 6.1$

Stature (cm) $\quad 172.0 \pm 4.3 \quad 172.0 \pm 4.3$

Body Mass (Kg) $73.1 \pm 14.6 \quad 71.4 \pm 14.5$

BMI $\left(\mathbf{K g} / \mathbf{m}^{2}\right) \quad 25.5 \pm 4.1 \quad 24.5 \pm 7.5$

Body Fat $(\mathbf{K g}) \quad 24.3 \pm 9.5 \quad 23.7 \pm 10.8$

$-2 \%$

$-3.8 ; 2.8$

$-4 \% \quad-1.6 ;-0.2$

$-3 \% \quad-7.3 ; 0.39$

LBM (Kg)

$47.6 \pm 7.7$

$45.0 \pm 6.4$

$-3.99 ;-1.14$

GM ACSA $\left(\mathrm{cm}^{2}\right)$

$23.3 \pm 16.5$

$21.4 \pm 16.3$

$-5 \%$ *

$-3.43 ;-0.49$

PFMVC (N.m)

$16.7 \pm 6.8$

$13.6 \pm 6.3$

$-4.79 ;-1.49$

KEMVC (N.m)

$12.6 \pm 8.8$

$10.8 \pm 7.0$

$-19 \% *$

$-3.16 ;-0.31$

SB\%

$96.4 \pm 4.5$

$98.5 \pm 0.02$

$-14 \% *$

$-0.32 ; 4.54$

TPA $^{\text {mins }}$

$13.5 \pm 16.1$

$7.17 \pm 8.9$

$-47 \%$

$-14 ; 1.7$

559

Table 1. One year changes in MD strength, physical activity and function. All data presented and Mean \pm SD, except for Functional status which is presented as Median (Range), Respiratory Support, Ambulatory Status and PEG are presented as absolute. DMD = Duchenne Muscular Dystrophy; 95\% CI = 95\% Confidence Intervals PEG = Percutaneous endoscopic gastrostomy; PFMVC = Plantar-Flexion Maximum Voluntary Contraction; KEMVC $=$ Knee Extension maximum Voluntary Contraction; SB $\%=$ Sedentary Behaviour $\%$; TPA $^{\text {mins }}=$ Minutes of Total Physical Activity; $\mathrm{m}=$ metres; $\mathrm{s}=$ seconds; $\uparrow$ Ambulant BMD only $(\mathrm{n}=8)$; ${ }^{*}$ denotes significant changes from baseline.

Table 2. 12 Month changes in body composition, muscle size, lower limb strength and physical activity in Adults with BMD.

\begin{tabular}{|c|c|c|c|c|}
\hline \multicolumn{5}{|c|}{ BMD } \\
\hline & Baseline & 12 Months & $\%$ Change & $95 \%$ CI \\
\hline $\mathbf{N}$ & & & & \\
\hline $\begin{array}{l}\text { Functional Status } \\
\text { Ambulatory Status }\end{array}$ & $3.5(1-7)$ & $3.5(1-7)$ & - & - \\
\hline No Walking Support & 6 & 6 & - & - \\
\hline Walking Support & 3 & 2 & - & - \\
\hline Manual Wheelchair & 1 & 2 & - & - \\
\hline Electric Wheelchair & 2 & 2 & - & - \\
\hline $\begin{array}{c}\text { Respiratory } \\
\text { Support }\end{array}$ & $0 / 12$ & $0 / 12$ & - & - \\
\hline Night-time only & - & - & - & - \\
\hline $24 / 7$ & - & - & - & - \\
\hline PEG & $0 / 12$ & $0 / 12$ & - & - \\
\hline Age (years) & $44.1 \pm 12.6$ & $45.1 \pm 12.6$ & - & - \\
\hline Stature (cm) & $178.9 \pm 6.2$ & $178.9 \pm 6.2$ & - & - \\
\hline Body Mass (Kg) & $84.4 \pm 15.1$ & $85.1 \pm 16.4$ & $0 \%$ & $-1.22 ; 2.64$ \\
\hline BMI $\left(\mathrm{Kg} / \mathbf{m}^{2}\right)$ & $26.4 \pm 4.9$ & $26.6 \pm 5.4$ & $0 \%$ & $-0.38 ; 0.84$ \\
\hline Body Fat (Kg) & $25.1 \pm 8.8$ & $26.3 \pm 8.9$ & $4 \% *$ & $0.20 ; 2.19$ \\
\hline
\end{tabular}




\begin{tabular}{ccccc} 
LBM (Kg) & $59.3 \pm 7.8$ & $58.8 \pm 8.1$ & $-1 \%$ & $-2.05 ; 1.08$ \\
Ambulatory & $9 / 12$ & $8 / 12$ & - & - \\
GM ACSA $\left(\mathbf{c m}^{2}\right)$ & $29.7 \pm 18.4$ & $26.6 \pm 14.4$ & $-10 \%$ & $-6.0 ;-0.11$ \\
PFMVC (N.m) & $35.7 \pm 11.3$ & $33.2 \pm 12.2$ & $-7 \%$ & $-6.01 ; 1.08$ \\
KEMVC (N.m) & $97.7 \pm 64.3$ & $83.9 \pm 56.2$ & $14 \%$ & $-24.8 ;-2.6$ \\
SB\% & $83.4 \pm 7.2$ & $83.9 \pm 6.3$ & $0 \%$ & $-4 ; 5$ \\
TPA & $123.1 \pm 57.6$ & $120.4 \pm 50.7$ & $-2 \%$ & $-17.2 ; 70.5$ \\
10m Walk (s) $\dagger$ & $11.0 \pm 2.9$ & $12.7 \pm 3.9$ & $15 \% *$ & $1.4 ; 3.4$ \\
\hline
\end{tabular}

Table 2. One year changes in MD strength, physical activity and function. All data presented and Mean \pm SD, except for

568 Functional status which is presented as Median (Range), Respiratory Support, Ambulatory Status and PEG which are presented 569 as absolute. BMD = Beckers Muscular Dystrophy; 95\% CI = 95\% Confidence Intervals; PEG = Percutaneous endoscopic 570 gastrostomy; PFMVC $=$ Plantar-Flexion Maximum Voluntary Contraction; KEMVC $=$ Knee Extension maximum Voluntary 571 Contraction; SB\% = Sedentary Behaviour \%; $\mathrm{TPA}^{\text {mins }}=$ Minutes of Total Physical Activity; $\mathrm{m}=$ metres; $\mathrm{s}=$ seconds; $\uparrow$ 572 Ambulant BMD only $(\mathrm{n}=8) ;{ }^{*}$ denotes significant changes from baseline.

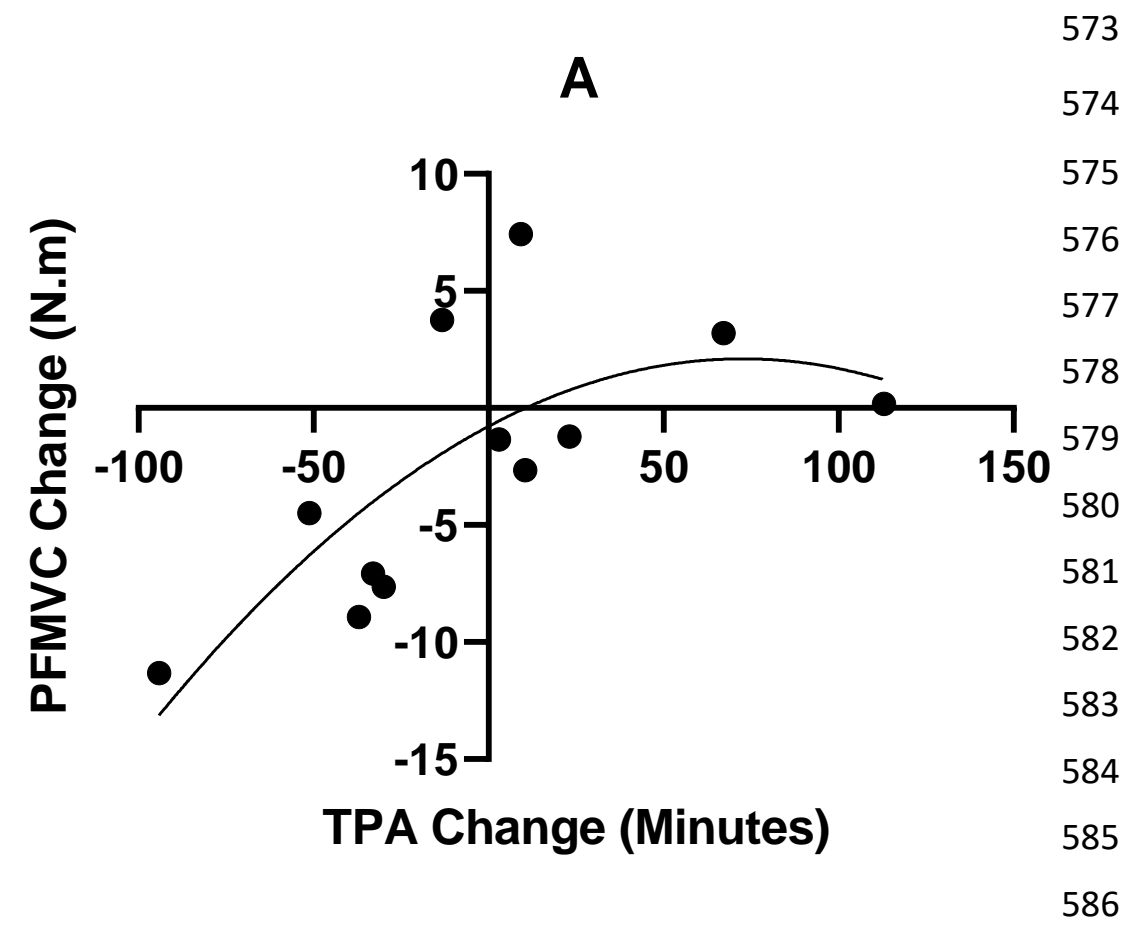




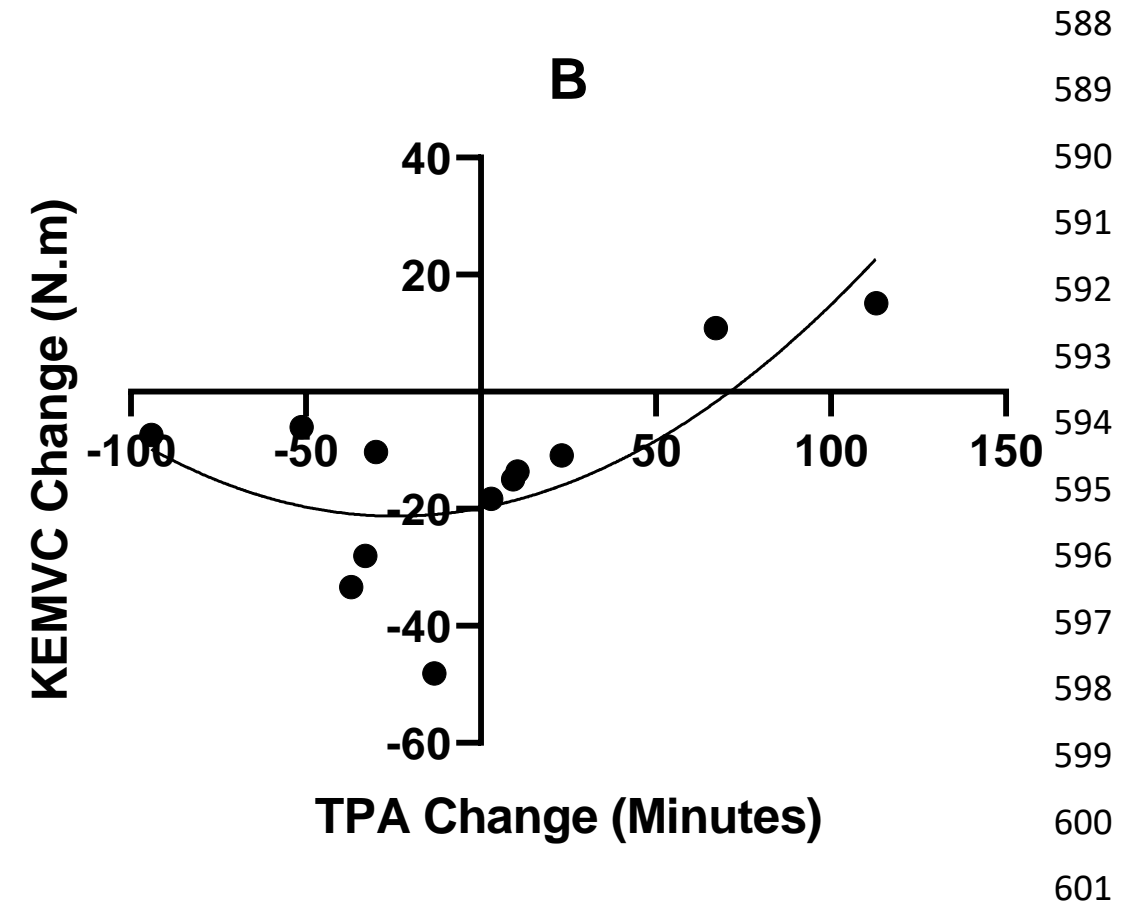

602 Figure 1. BMD strength change and physical activity change relationships A. PFMVC change and TPA ${ }^{\text {mins }}$ change in BMD B. 603 KEMVC change and TPA ${ }^{\text {mins }}$ change in BMD. PFMVC = Plantar Flexion Maximal Voluntary Contraction, N.m = Newton 604 Metres, TPA = Total Physical Activity, KEMVC $=$ Knee Extension Maximal Voluntary Contraction. 